Somnologie $2016 \cdot 20: 2-3$

DOI 10.1007/s11818-016-0046-8

Online publiziert: 23. Februar 2016

๑) Springer-Verlag Berlin Heidelberg 2016

CrossMark

\author{
Thomas Penzel $\cdot$ Michael Arzt ${ }^{2}$ \\ 'Schlafmedizinisches Zentrum, Charité Campus Mitte, Berlin, Deutschland \\ ${ }^{2}$ Schlafmedizinisches Zentrum der Klinik und Poliklinik für Innere Medizin II, Pneumologie, \\ Universitätsklinikum Regensburg, Regensburg, Deutschland
}

\title{
Internationale Forschung und nationale Weiterbildung
}

Mit dem Einstieg des neuen Herausge-

Mit dem Jahreswechsel 2015 auf 2016 erfahren wir mehrere größere Änderungen für die Somnologie. Der langjährige Mitherausgeber der Somnologie, Prof. Dr. Geert Mayer, legt dieses Amt nieder, um sich anderen Aufgaben der Schlafmedizin in Deutschland $\mathrm{zu}$ widmen. Seine aktuelle und sehr zeitaufwendige Aufgabe ist die Aktualisierung der Leitlinie „Schlafbezogene Atmungsstörungen“, die noch in diesem Jahr in der Somnologie erscheinen wird. Sein Nachfolger im Amt der Herausgeberschaft ist Prof. Dr. Michael Arzt, der seit vielen Jahren das Schlaflabor an der Universität Regensburg leitet und international wie national als engagierter Schlafforscher und Schlafmediziner bekannt ist. Gemeinsam danken wir Geert Mayer für seine engagierte und langjährige Tätigkeit und seine Aktivitäten für das Einwerben von Schwerpunktheften und Autoren für unsere Zeitschrift Somnologie. Im Rahmen der DGSMJahrestagung 2015 in Mainz wurde ihm auch von der Mitgliederversammlung offiziell Dank ausgesprochen, er wurde zusätzlich im Rahmen des Gesellschaftsabends geehrt. Ein besonderes Anliegen war Geert Mayer immer der Beitrag der neurologischen Schlafmedizin und das Unterstützen des wissenschaftlichen Nachwuchses. Auch weiterhin organisiert Geert Mayer Workshops für Nachwuchswissenschaftler mit Teilnehmern aus deutschen Schlaflaboren und internationalen Sprechern. Für die Somnologie sind daraus immer wieder spannende und ganz aktuelle Beiträge aus der angewandten und Grundlagenforschung erwachsen. In diesem Sinne wollen wir die Somnologie weiterführen. bers Michael Arzt führen wir eine Reihe von Neuerungen ein. Eine wichtige und langfristig bedeutsame Änderung ist die Einrichtung einer CME-Rubrik in der Somnologie. Die CME-Rubrik stellt eine wichtige Bereicherung für die Somnologie zur Unterstützung der Weiterbildung dar. Das auf den Jahrestagungen durchgeführte Kolleg zur Weiterbildung in der Schlafmedizin wird hierdurch auch in schriftlicher Form ergänzt und ermöglichen den Erwerb von CME-Punkten. Für die CME-Rubrik konnten wir als verantwortliche Herausgeber Prof. Richard Schulz und Dr. Holger Hein gewinnen. Damit haben wir hierfür zwei Personen, die sowohl in der Forschung als auch in der Praxis ausgewiesene Experten sind. Sie werden in den kommenden Monaten Kollegen ansprechen, um CME-Beträge für die Somnologie zu erbitten. In jedem Somnologie-Heft soll in Zukunft ein CME-Beitrag erscheinen. Der erste CME-Beitrag ist im Entstehen und wird im dritten Heft 2016 erscheinen.

Als weitere Neuerung wollen wir das Einreichen und Begutachten der Manuskripte erleichtern. Dazu werden wir nunmehr auch für die Somnologie das elektronische System zum Verwalten von Manuskripten, den Editorial Manager, einsetzen. Viele Autoren und Gutachter kennen es schon von anderen Fachzeitschriften. Im Hintergrund wird dieses System von uns Herausgebern und von Frau W. Gorgolik als Assistenz der Schriftleitung betreut. Für die Autoren und Gutachter bedeutet diese Umstellung in der Regel eine Vereinfachung auf ein bereits bekanntes System. Bei inhaltlichen Fragen können Sie sich, wie gehabt, an uns wenden und für technische Fragen steht auch der SpringerVerlag wie bisher hilfreich zur Seite.

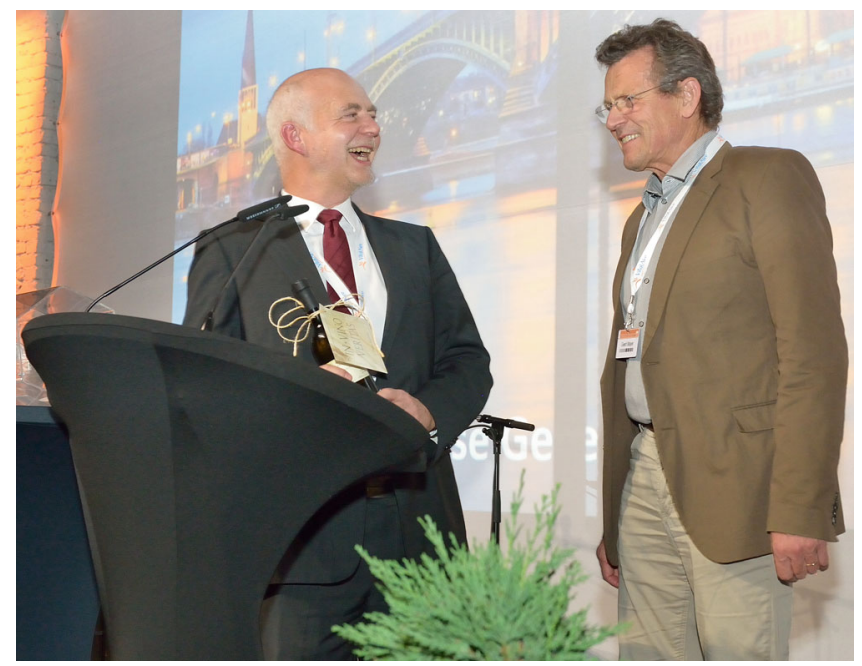

Herr Prof. Mayer bei seiner Verabschiedung (mit Herrn Prof. Penzel) 
Auch für dieses Jahr sind wieder spannende und aktuelle Schwerpunkthefte geplant. Zusätzlich nehmen wir gerne Studien, Übersichtsarbeiten, Fallberichte und weitere interessante Beiträge entgegen.

Viel Erfolg für das Jahr wünschen Ihnen Ihre Somnologie-Herausgeber,

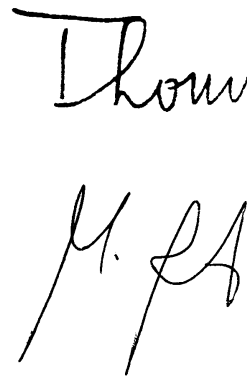

Thomas Penzel und Michael Arzt

\section{Korrespondenzadresse}

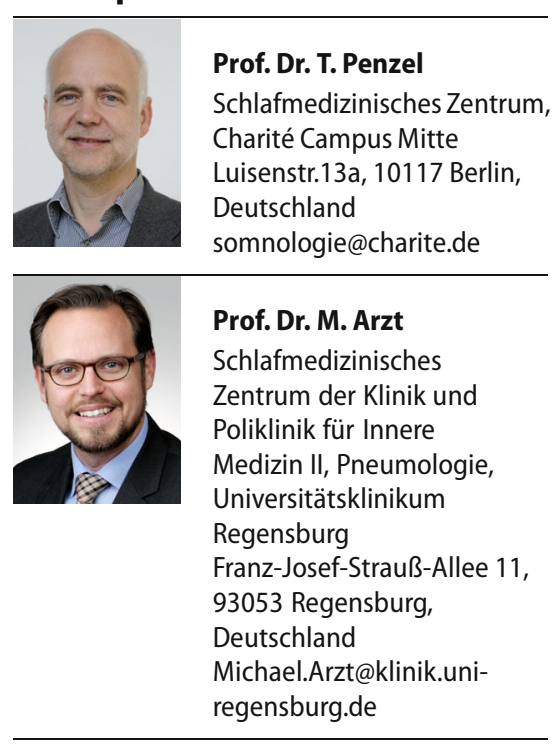

Interessenkonflikt. T. Penzel und M. Arzt geben an, dass kein Interessenkonflikt besteht. 